\title{
Genetic inactivation of IL-1 signaling enhances atherosclerotic plaque instability and reduces outward vessel remodeling in advanced atherosclerosis in mice
}

\author{
Matthew R. Alexander, ${ }^{1,2}$ Christopher W. Moehle, ${ }^{1,2}$ Jason L. Johnson, ${ }^{3}$ Zhengyu Yang, ${ }^{4}$ \\ Jae K. Lee, ${ }^{4}$ Christopher L. Jackson, ${ }^{3}$ and Gary K. Owens ${ }^{1,2}$ \\ 1Department of Molecular Physiology and Biological Physics and ${ }^{2}$ Robert M. Berne Cardiovascular Research Center, University of Virginia, \\ Charlottesville, Virginia, USA. ${ }^{3}$ Bristol Heart Institute, University of Bristol, Bristol, United Kingdom. ${ }^{4}$ Department of Public Health Sciences, \\ University of Virginia, Charlottesville, Virginia, USA.
}

\begin{abstract}
Clinical complications of atherosclerosis arise primarily as a result of luminal obstruction due to atherosclerotic plaque growth, with inadequate outward vessel remodeling and plaque destabilization leading to rupture. IL-1 is a proinflammatory cytokine that promotes atherogenesis in animal models, but its role in plaque destabilization and outward vessel remodeling is unclear. The studies presented herein show that advanced

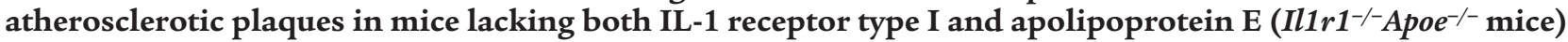
unexpectedly exhibited multiple features of plaque instability as compared with those of $I l 1 \mathrm{rI}^{+/+} \mathrm{Apoe}^{-/-}$mice. These features included reduced plaque SMC content and coverage, reduced plaque collagen content, and increased intraplaque hemorrhage. In addition, the brachiocephalic arteries of $I l 1 \mathrm{r1}^{-/-} \mathrm{Apoe}^{-/-}$mice exhibited no difference in plaque size, but reduced vessel area and lumen size relative to controls, demonstrating a reduction in outward vessel remodeling. Interestingly, expression of MMP3 was dramatically reduced within the plaque and vessel wall of $\mathrm{Il1} \mathrm{r1}^{-/-} \mathrm{Apoe} \mathrm{e}^{-/-}$mice, and $\mathrm{Mmp3}^{-/-} \mathrm{Apoe}^{-/-}$mice showed defective outward vessel remodeling compared with controls. In addition, MMP3 was required for IL-1-induced SMC invasion of Matrigel in vitro. Taken together, these results show that IL-1 signaling plays a surprising dual protective role in advanced atherosclerosis by promoting outward vessel remodeling and enhancing features of plaque stability, at least in part through MMP3-dependent mechanisms.
\end{abstract}

\section{Introduction}

Atherosclerosis is a chronic disease affecting large arteries that involves the formation of plaques containing inflammatory and vascular cells, extracellular matrix, and lipid (1). Clinical complications of atherosclerosis arise through obstruction of the arterial lumen, leading to insufficient oxygen supply for dependent tissues. Most of the morbidity and mortality associated with atherosclerosis occur due to disease in the coronary circulation of the heart, where luminal obstruction occurs through 2 primary mechanisms: (a) plaque growth with inadequate outward vessel remodeling, leading to vessel stenosis, and (b) formation of unstable plaques that acutely rupture, precipitating occlusive thrombus formation (2). Despite extensive research, there are fundamental gaps in our knowledge of these processes, and a tremendous need exists for better understanding the pathophysiology of atherosclerotic vascular disease in order to develop new therapeutic strategies to prevent its clinical complications.

Atherosclerosis is an inflammatory disease characterized by recruitment of numerous circulating inflammatory cells, including monocytes/macrophages, $\mathrm{T}$ lymphocytes, and neutrophils (3). Proinflammatory cytokines are thought to be detrimental in

Conflict of interest: Gary K. Owens is a shareholder and chief scientific officer of NanoMedical Systems Inc., which develops stent technology unrelated to these studies. Gary K. Owens and Christopher L. Jackson have independently received research funding from AstraZeneca Pharmaceutical Inc. for studies separate from those reported in this manuscript. Jae K. Lee reports ownership of Key Genomics Inc. Citation for this article: J Clin Invest. 2012;122(1):70-79. doi:10.1172/JCI43713. atherosclerosis due in large part to their role in promoting atherosclerotic plaque formation by enhancing leukocyte recruitment and activation $(4,5)$. However, the degree of outward remodeling of atherosclerotic vessels better determines lumen size than does plaque area $(6,7)$, and plaque composition is a better determinant of plaque stability and propensity to rupture than is the size of the plaque (8-12). Evidence suggests that proinflammatory cytokines also promote features of atherosclerotic plaque destabilization, as inhibition of proinflammatory cytokines such as IL-18 (13), monocyte chemoattractant protein-1 (14), and IFN- $\gamma(15)$ in atheroprone mice promotes features of plaque stability such as increased SMC and collagen content. However, to date, only a limited number of inflammatory cytokines have been tested for causative roles in regulating features of plaque stability. Additionally, the potential role of inflammatory cytokines in outward vessel remodeling and luminal narrowing in atherosclerosis is virtually unknown.

IL-1 is a proinflammatory cytokine that plays a central role in mediating innate and adaptive immune responses to multiple chemical, infectious, and mechanical insults (16). The term IL-1 refers to 2 cytokines, IL- $1 \alpha$ and IL-1 $\beta$, which signal exclusively through a common receptor, IL-1 receptor type I (gene: Il1r1; protein: IL-1R1) (17). IL-1 $\alpha$ and IL-1 $\beta$ are found in human atherosclerotic plaques, where they are released primarily by endothelial cells and macrophages $(18,19)$. Genetic inactivation and pharmacological inhibition of IL-1 $\alpha$, IL-1 $\beta$, and IL-1R1 have been shown to decrease atherosclerotic plaque formation within the aortic root and thoracoabdominal aorta in mice (20-25), demonstrating a pro- 
A

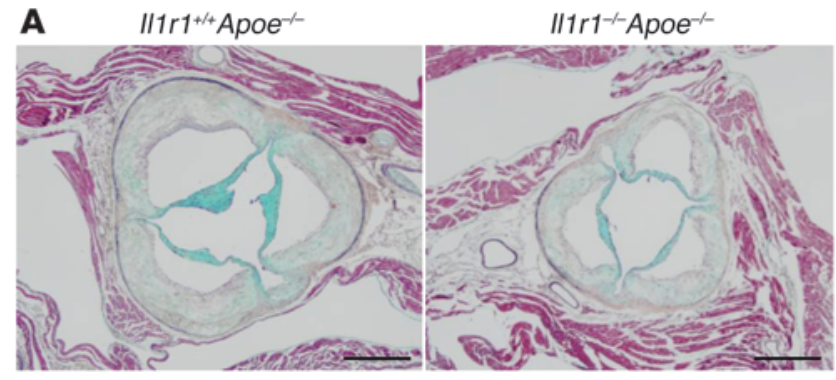

B

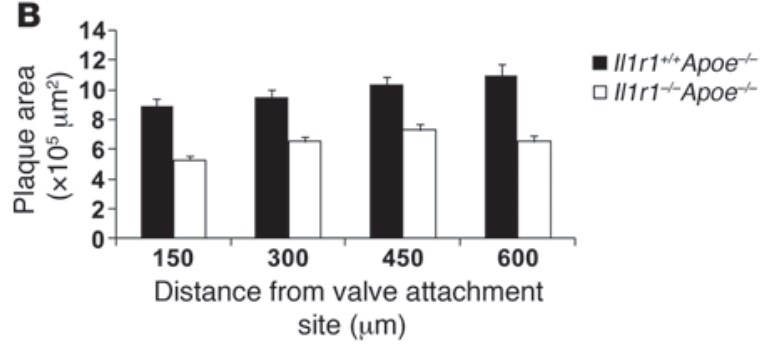

Figure 1

IL-1R1 deficiency reduces advanced atherosclerotic plaque size at the aortic root. (A) Representative images of Movat-stained aortic sections of $/ 11 r 1^{-/-} \mathrm{Apoe}^{-/-}$and $/ 11 \mathrm{r}^{+{ }^{++}} \mathrm{Apoe^{-/ }}$ mice after 27-30 weeks of high-fat diet feeding. Scale bars: $500 \mu \mathrm{m}$. (B) Quantification of total atherosclerotic plaque area within the aortic root of $/ 11 \mathrm{r}^{+/+} \mathrm{Apoe^{-/- }}$ and $1 / 1 \mathrm{r}^{-1-} \mathrm{Apoe}^{-/-}$mice at $150-\mu \mathrm{m}$ intervals from the aortic valve attachment site. ${ }^{*} P<0.001$ for difference between genotypes by ScheirerRay-Hare test. $n=13, \| / 1 r 1^{+/+} A p o e^{-/-} ; n=12, I / 1 r 1^{-/-} A p o e^{-/-}$. Data represent mean \pm SEM.

atherogenic role for IL-1 in promoting atherosclerotic lesion formation. However, these studies have focused almost exclusively on the effects of IL-1 on lesion size and the extent of lesion formation, and a comprehensive analysis of the role of IL- 1 in regulating features of plaque stability and outward vessel remodeling in advanced atherosclerosis has not been performed. Genetic inactivation of the endogenous inhibitor of IL-1 signaling, IL-1 receptor antagonist (IL-1RN), in advanced lesions of atheroprone mice has demonstrated increased plaque macrophage content and reduced SMC content, consistent with IL-1RN playing a beneficial role in regulating features of plaque stability (26). However, IL-1RN deficiency likely results in supraphysiological levels of IL-1 activity, IL-1RN has IL-1R1-independent effects (27), and indices of plaque stability used in this study were limited to assessing macrophage and SMC content. Because of this, further studies of the role of IL-1 signaling in advanced atherosclerosis are needed, particularly in light of ongoing major clinical trials in humans testing the role of IL- 1 inhibition in the clinical complications of atherosclerosis (28).

The present studies were initiated with a focus on testing the hypothesis that IL-1 promotes formation of an unstable plaque phenotype with impaired outward vessel remodeling and enhanced luminal narrowing in advanced atherosclerosis in the apolipoprotein E-deficient (apoE-deficient) (gene: Apoe; protein: APOE) mouse. To our surprise, results showed just the opposite. Inactivation of IL-1 signaling through loss of the IL-1R1 in Apoe-knockout mice resulted in enhancement of multiple indices of atherosclerotic plaque instability, including reduced plaque SMC content and coverage, reduced plaque collagen content, and increased intraplaque hemorrhage as well as impaired outward vessel remod- eling, leading to reduced lumen size. We also provide evidence that IL-1 signaling promotes expression of an extracellular protease, MMP3, which enhances outward vessel remodeling in atherosclerosis and mediates IL-1-induced migration of cultured SMCs. Taken together, these results indicate that IL-1 plays a surprising dual protective role in atherosclerosis by enhancing features of plaque stability and promoting outward vessel remodeling at least in part through enhancing MMP3 production.

\section{Results}

IL-1R1 deficiency reduces atherosclerotic plaque size at the aortic root. To determine the role of IL-1 in advanced atherosclerosis, mice lacking both IL-1 receptor type I and apoE (Il1r1-/-Apoe-/-) and singleknockout controls $\left(I l 1 r 1^{+/+} A p o e^{-/-}\right)$were fed a high-fat diet for 27-30 weeks to generate advanced atherosclerotic lesions. Upon completion of high-fat diet feeding, Illr1-/-Apoe $e^{-/}$mice did not significantly differ from control mice in metabolic measures, such as body weight, plasma cholesterol, or plasma triglyceride levels (Supplemental Figure 1; supplemental material available online with this article; doi:10.1172/JCI43713DS1). However, Illr1-/Apoe ${ }^{-/}$mice exhibited significantly reduced plaque size across 4 locations at the aortic root (Figure 1). These results are consistent with previous studies demonstrating a proatherogenic role for IL-1 signaling in aortic root atherosclerosis $(20,22,23,25,29)$.

IL-1R1 deficiency reduces outward remodeling of atherosclerotic vessels. In addition to the aortic root, we also tested the effect of IL-1R1 deficiency on atherosclerosis at the brachiocephalic artery, which is a site of advanced plaque formation in mice $(30,31)$. Interestingly, atherosclerotic plaque size within brachiocephalic arteries of Ill $\mathrm{r1}^{-/-} \mathrm{Apoe}^{-/-}$mice exhibited a trend toward a reduction, but did not significantly differ from those of $I l 1 r 1^{+/+} A_{p o e^{-/-}}$controls over 5 locations across the artery $(n=12-14$ mice per group; $P=0.07$ ) (Figure 2, A and B). However, the area of the brachiocephalic artery within the internal elastic lamina (IEL) was significantly reduced in Il1r1-/-Apoe ${ }^{-/-}$mice relative to $\mathrm{IlIrI}^{+/+} \mathrm{Apoe}^{-/-}$controls (Figure 2, A and C). In addition, Ill $\mathrm{rI}^{-/-} \mathrm{Apoe^{-/- }}$ mice exhibited a greater than $50 \%$ decrease in lumen area across the 5 brachiocephalic artery locations compared with $I l 1 r 1^{+/+}$Apoe $^{-/-}$control mice (Figure 2, A and D). These results demonstrate that, although IL-1R1 deficiency does not significantly alter plaque size at the brachiocephalic artery, inactivation of IL-1 signaling results in a reduction in compensatory outward vessel remodeling, leading to reduced lumen size.

Although morphometric analysis of vessel and lumen size within the aortic root can be complicated by the presence of valves, previous studies have measured the length of the IEL within each aortic sinus to calculate vessel area using the sum of these lengths as the circumference of a circle (32). Using these methods at the aortic root, results revealed that $I l 1 r 1^{-/-}$Apoe $e^{-/}$mice exhibited reduced vessel size relative to controls (Supplemental Figure 2A). Although this could be secondary to the reduced plaque size in these animals (Figure 1), lumen size at the aortic roots in Illr1-/-Apoe ${ }^{-/-}$mice was also significantly reduced relative to that in control mice (Supplemental Figure 2B). These results demonstrate a deficit in compensatory outward vessel remodeling at the aortic root in IL-1R1-deficient mice, which is consistent with the observed reduction in outward vessel remodeling at the brachiocephalic artery in IL-1R1-deficient mice. Taken together, these results suggest that IL-1 plays an unexpected beneficial role in advanced atherosclerosis by promoting outward vessel remodeling to limit narrowing of the vessel lumen. 

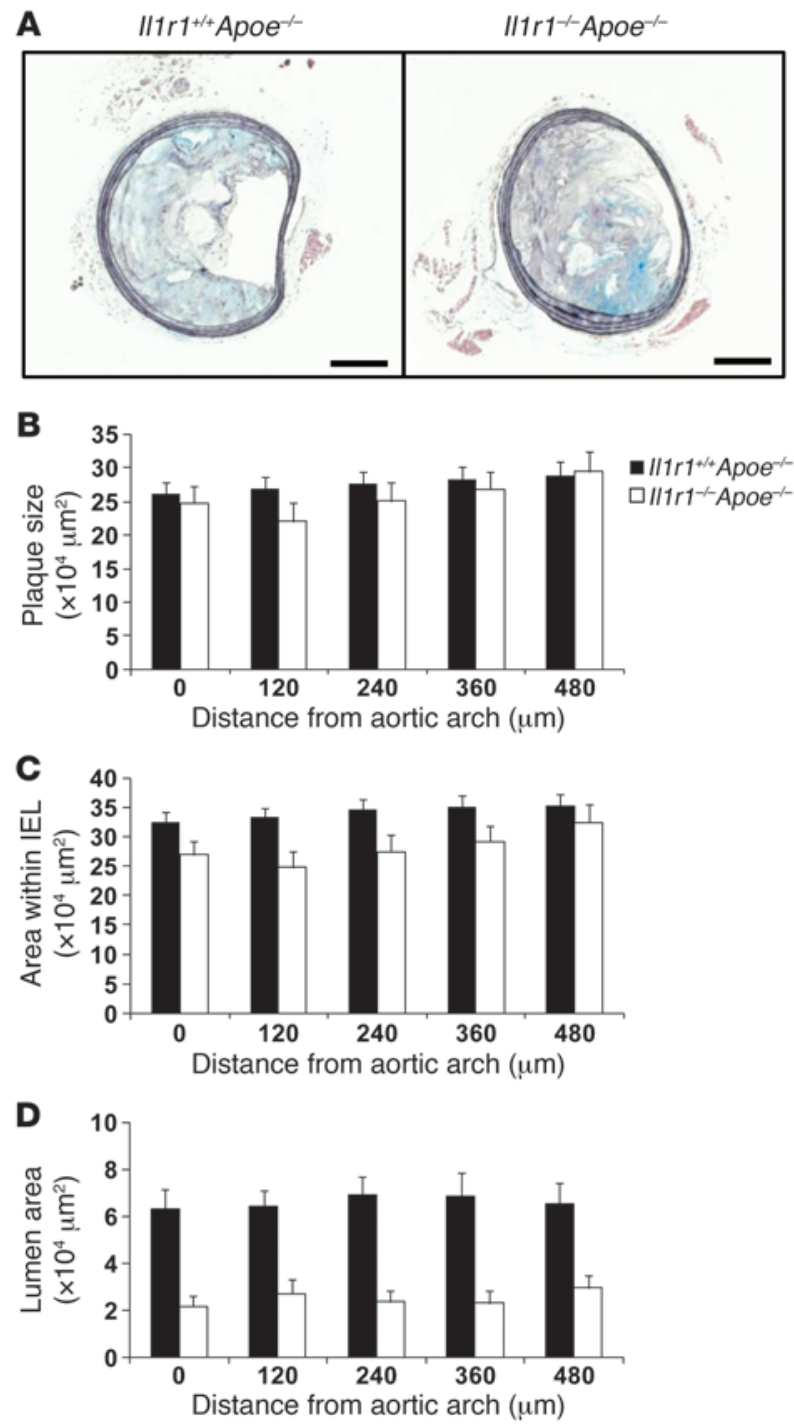

IL-1R1 deficiency enhances features of atherosclerotic plaque instability. To determine whether IL-1R1 deficiency alters features of atherosclerotic plaque stability, plaques were analyzed at the brachiocephalic artery for features that both associate with and contribute to plaque instability in humans, such as decreased plaque collagen content (33-36) and decreased SMC coverage of the plaque at the fibrous cap region as well as decreased total SMC content within the plaque (37-39), increased plaque macrophage content $(37,39)$, and increased intraplaque hemorrhage $(40,41)$. Since IL- 1 is a proinflammatory cytokine, it was our original hypothesis that loss of IL-1 signaling would promote features of plaque stability similarly to proinflammatory cytokines tested previously (13-15). Surprisingly, results showed just the opposite. First, plaque collagen content, which plays an important structural role in stabilizing plaques and is decreased in unstable human lesions (33-36), was decreased in $I l 1 r 1^{-/-}$Apoe ${ }^{-/-}$relative to $I l 1 r 1^{+/+} A_{p o e^{-/-}}$control mice (Figure 3, A and F). Second, both SMC coverage of the luminal surface of the plaque in the region of the fibrous cap and total plaque SMC content were significantly decreased in $I l 1 r 1^{-/-}$Apoe $^{-/-}$mice relative to controls (Figure 3, B, G, and H), consistent with features of unstable plaques in humans (37-39). Third, macrophage con-

\section{Figure 2}

IL-1R1 deficiency reduces compensatory outward remodeling of atherosclerotic brachiocephalic arteries. (A) Movat staining of representative brachiocephalic arteries of $/ 11 \mathrm{r}^{-{ }^{-/}}$Apoe $\mathrm{e}^{-/-}$and $/ 11 \mathrm{r} 1^{+/+} \mathrm{Apoe^{-/ }}$ mice. Scale bars: $200 \mu \mathrm{m}$. (B-D) Atherosclerotic plaque area (B), vessel area within the IEL $(\mathbf{C})$, and lumen area, $P<0.001$ for difference between genotypes by 2-way ANOVA, (D) at multiple locations along the brachiocephalic arteries of $I / 1 \mathrm{rr}^{-/-} \mathrm{Apoe}^{-/}$and $/ 11 \mathrm{r}^{+/+} \mathrm{Apoe}^{-/-}$mice, $P<0.001$ for difference between genotypes by 2-way ANOVA after square root transformation. $n=14, \| / 1 \mathrm{r}^{+/+} \mathrm{Apoe}^{-/-} ; n=12, I / 1 \mathrm{r}^{-/-} \mathrm{Apoe}^{-/}$. Data in $\mathbf{B}-\mathbf{D}$ represent mean \pm SEM.

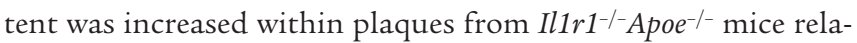
tive to controls (Figure 3, C and I), which is also consistent with increased plaque instability $(37,39)$. Finally, intraplaque hemorrhage, defined as the presence of rbc within the plaque, was also significantly increased in $I l 1 r 1^{-/-} A p o e^{-/-}$relative to $I l 1 r 1^{+/+} A p o e^{-/-}$ mouse lesions (Figure 3, D, E, and J). Although the source of rbc within Il1r1 $1^{--}$Apoe $^{-/-}$plaques is unclear, increased presence of $\mathrm{rbc}$ within plaques is associated with an unstable plaque phenotype in humans and also may directly contribute to plaque instability owing to the high cholesterol content of rbc (40-42). Collectively, these results demonstrate that inactivation of IL-1 signaling in advanced atherosclerosis surprisingly promotes features of an unstable plaque phenotype. Consistent with this, atherosclerotic plaques at the aortic root also exhibited decreased plaque collagen content, reduced plaque SMC coverage, and increased intraplaque hemorrhage in $\mathrm{Il1} \mathrm{rI}^{-/-} \mathrm{Apoe^{-/ }}$ mice relative to controls (Supplemental Figure 3). Taken together, these data provide evidence that IL-1 signaling plays an unexpected protective role in advanced atherosclerosis by enhancing multiple features of atherosclerotic plaque stability at both the brachiocephalic artery and aortic root.

IL-1R1 deficiency reduces MMP3 expression within the brachiocephalic artery plaque and vessel wall. We next sought to determine the mechanisms by which loss of IL-1 signaling results in reduced outward vessel remodeling and enhanced features of plaque instability. Evidence suggests that SMCs play an important role in promoting outward remodeling of atherosclerotic vessels (43) as well as in enhancing atherosclerotic plaque stability $(44,45)$. Matrix metalloproteinases are secreted proteases that play an important role in promoting outward vessel remodeling and regulating atherosclerotic plaque stability, at least in part through their ability to degrade and remodel the extracellular matrix $(7,46)$. Previous studies have demonstrated that IL-1 promotes expression and activity of multiple MMPs in cultured SMCs $(47,48)$. In order to determine the full spectrum of MMPs induced by IL-1 in SMCs, we performed Affymetrix GeneChip microarray analysis on cultured SMCs stimulated with IL-1. IL-1 treatment of these cells induced the greatest increase ( $>6$-fold) in the expression of MMP3, MMP13, and MMP9 among all annotated MMPs (Supplemental Table 1 and see Supplemental Methods). Increases in MMP3, MMP9, and MMP13 expression were confirmed by realtime RT-PCR analysis (Supplemental Figure 4). Interestingly,

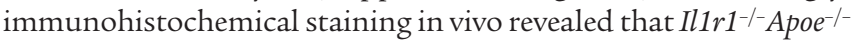
mice exhibited markedly decreased expression of MMP3 within the brachiocephalic artery plaque and vessel media relative to Illr $1^{+/+}$Apoe $e^{-/-}$controls (Figure 4). However, MMP13 levels did not differ within the plaque or vessel wall (Supplemental Figure 5, A, $\mathrm{C}$, and D). MMP9 levels were increased within the plaque in $\mathrm{Il1r1} \mathrm{r}^{-/}$ Apoe $e^{-/}$mice, and a nonsignificant trend existed for increased 

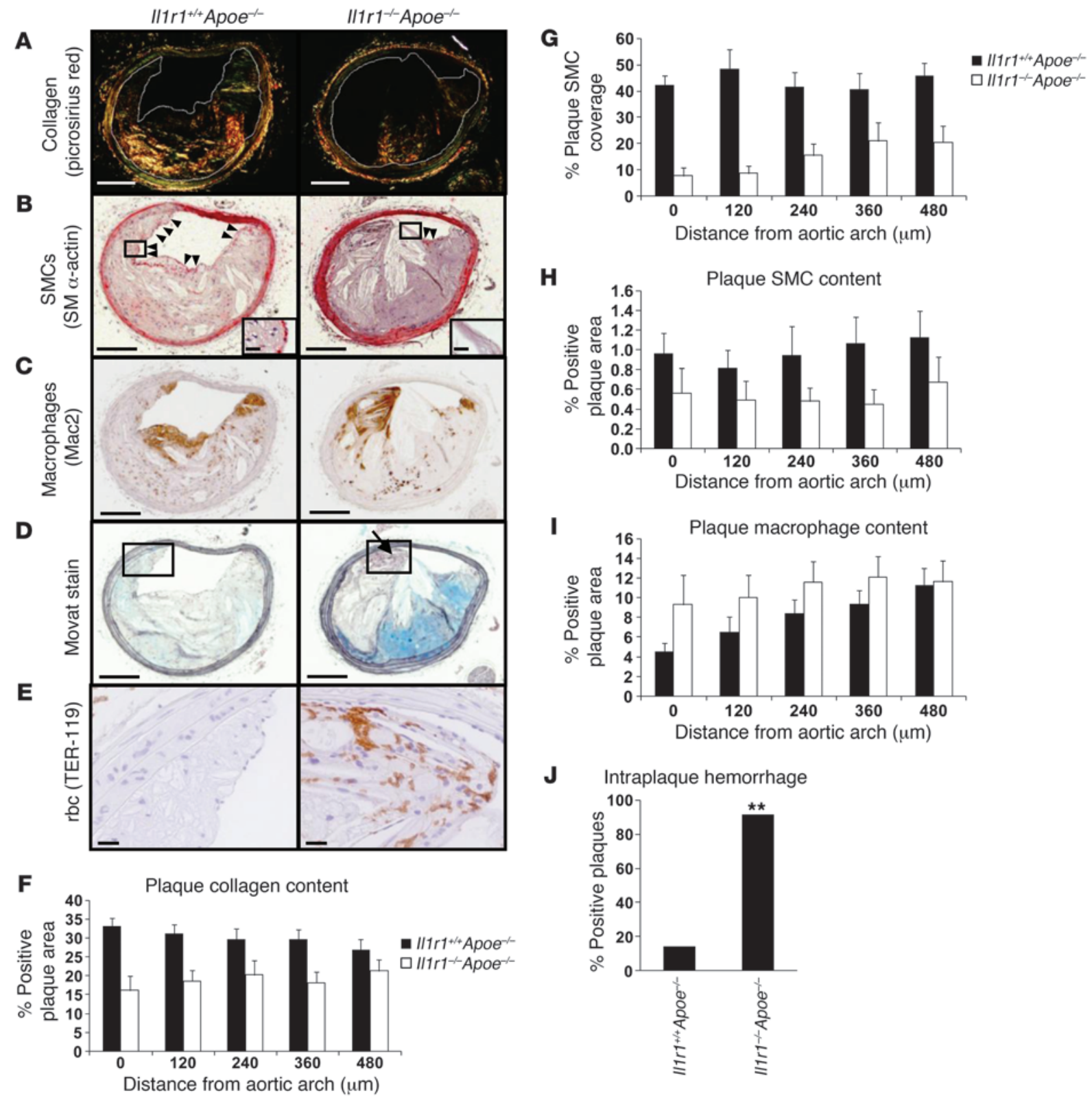

H

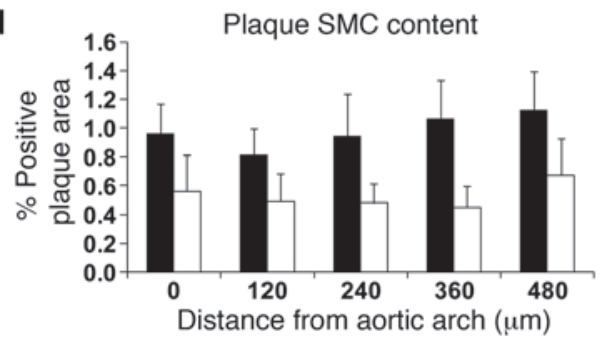

I

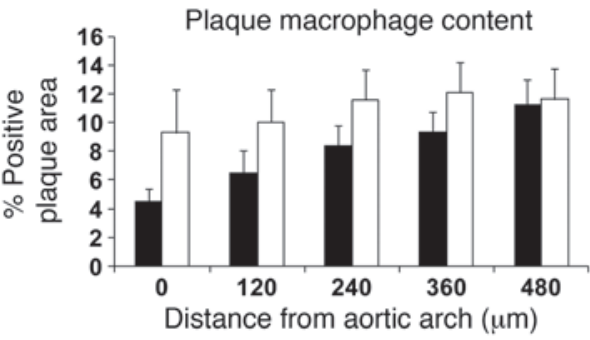

$\mathbf{J}$

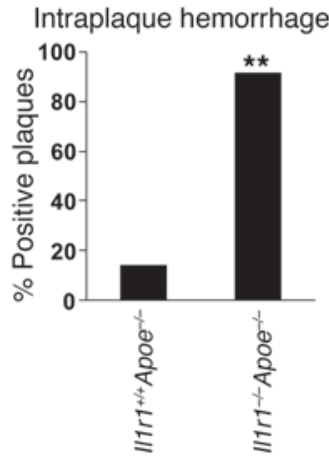

Figure 3

IL-1R1 deficiency decreases features of atherosclerotic plaque stability. (A-E) Representative images from brachiocephalic artery lesions of $\| / 1 \mathrm{r}^{+/+} \mathrm{Apoe}^{-/-}$and $/ 11 \mathrm{r} 1^{-/-}$Apoe $e^{-/-}$mice with (A) picrosirius red staining and polarized light microscopy for collagen detection, (B) SM $\alpha$-actin immunostaining for detecting SMCs on the plaque luminal surface (arrowheads) and total plaque SMC content, (C) Mac2 immunostaining for detection of plaque macrophages, (D) Movat staining for intraplaque rbc (arrow), and (E) immunostaining for the rbc marker TER-119 (magnified from boxed area in $\mathbf{D})$. ( $\mathbf{F}-\mathbf{J})$ Quantification of $(\mathbf{F})$ plaque collagen content based on picrosirius red staining, $P<0.001$ for difference of genotypes by 2-way ANOVA, (G) plaque SMC coverage based on SM a-actin staining, $P<0.001$ for difference of genotypes by the Scheirer-Ray-Hare test, (H) total plaque SMC content based on SM a-actin staining, $P<0.001$ for difference of genotypes by the Scheirer-Ray-Hare test (I) plaque macrophage content based on Mac2 staining, $P=0.01$ for difference of genotypes by 2-way ANOVA after log transformation, and $(\mathbf{J})$ the percentage of brachiocephalic arteries exhibiting intraplaque hemorrhage based on Movat and TER-119 staining, ${ }^{* *} P<0.01$ by Fisher's exact test. Data in F-I represent mean \pm SEM. $n=14, I / 1 r 1^{+/+} A p o e^{-/-} ; n=12, I / 1 r 1^{-1-} A p o e^{-/-}$. Scale bars: $200 \mu \mathrm{m}$ (A-D); $20 \mu \mathrm{m}$ (inset, B; E).

MMP9 levels within the media of the vessel $(P=0.1)$ (Supplemental Figure 5, B, E, and F), perhaps as a compensatory response to the markedly decreased MMP3 levels. Taken together, these results provide evidence that IL-1 plays an important role in the regulation of MMP3 production within atherosclerotic plaques and that MMP3 may mediate at least some of the observed effects of IL-1R1 deficiency in atherosclerosis.

MMP3 deficiency in Apoe-knockout mice reduces outward vessel remodeling. Although increased plaque MMP3 levels have been associated with outwardly remodeled atherosclerotic vessels in humans (49), 
A
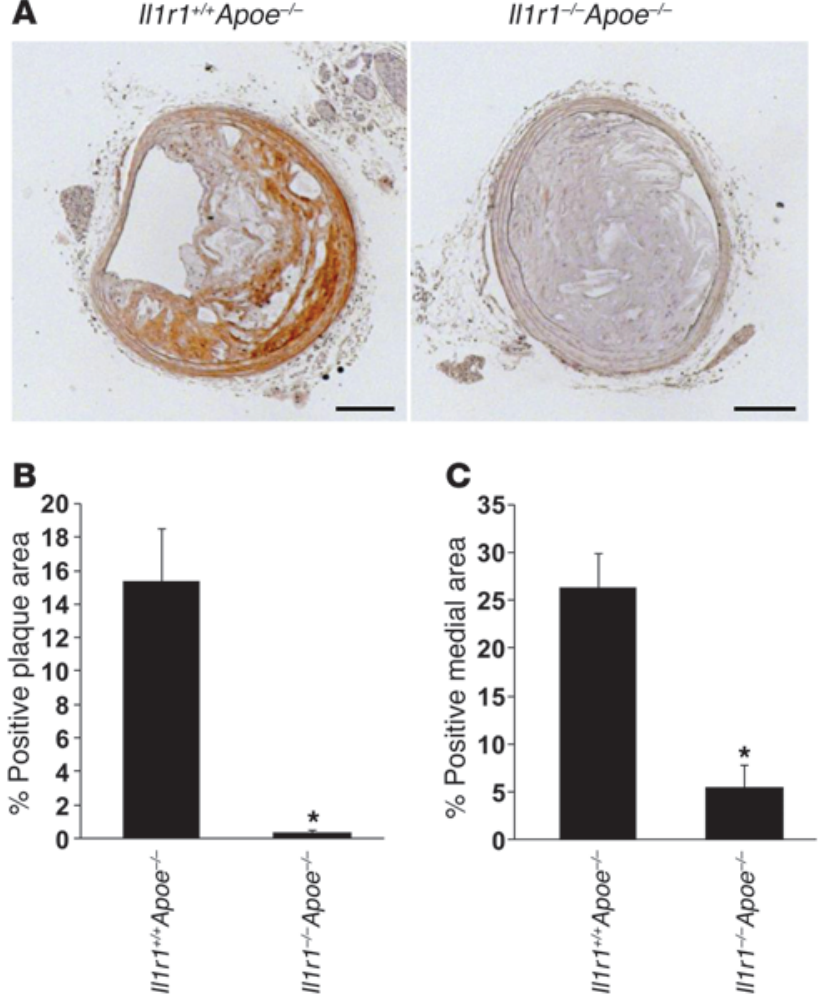

it is unclear whether MMP3 mediates outward vessel remodeling in atherosclerosis. In order to test this, we analyzed brachiocephalic artery lumen size after high-fat diet feeding in Apoe-knockout mice with and without genetic inactivation of MMP3 (designated $\mathrm{Mmp}^{3^{-/-}} \mathrm{Apoe}^{-/-}$and $\mathrm{Mmp}^{3^{+/+}} \mathrm{Apoe^{-/- }}$ mice, respectively) (50). $\mathrm{Mmp3}^{-/-} \mathrm{Apoe}^{-/-}$exhibit increased brachiocephalic artery lesion size relative to $\mathrm{Mmp3}^{+/+} A \mathrm{poe}^{-/-}$controls, as reported previously (50). The present studies extend those findings by demonstrating that brachiocephalic arteries of $\mathrm{Mmp}^{3^{-/-}} \mathrm{Apoe}^{-/-}$mice exhibited reduced lumen area, with no difference in vessel size relative to $\mathrm{Mmp}^{3^{+/+}} \mathrm{Apoe}^{-/-}$controls (Supplemental Figure 6). Thus, despite an increased lesion size in MMP3-deficient mice, these mice did not exhibit a compensatory increase in vessel size relative to controls, leading to a reduction in lumen area. These results suggest that MMP3 promotes outward remodeling of atherosclerotic vessels to limit luminal narrowing. Combined with the dramatic reductions in MMP3 levels in brachiocephalic arteries of $\mathrm{Illr}_{11^{-/}}$Apoe $\mathrm{e}^{-/-}$mice relative to controls (Figure 4), these results suggest that MMP3 may mediate the effects of IL-1 in promoting outward vessel remodeling in atherosclerosis.

MMP3 deficiency reduces IL-1-induced SMC migration across Matrigel. Although IL-1R1 deficiency reduced multiple features of atherosclerotic plaque stability, one of the largest differences was the reduction in plaque SMC content and coverage (Figure 3, A, G, and $\mathrm{H}$ ). SMC accumulation within atherosclerotic plaques as well as in the fibrous cap region at the plaque luminal surface involves, at least in part, SMC migration from the tunica media into the plaque and to the fibrous cap region at the luminal surface (51, 52). IL-1 has been shown to promote SMC migration through basement membrane matrices such as Matrigel (53); however, the mechanisms mediating IL-1-induced SMC migration through the matrix are unknown. Given the markedly reduced plaque and

\section{Figure 4}

IL-1R1 deficiency reduces MMP3 expression within the brachiocephalic artery plaque and vessel wall. (A) Representative images of MMP3 staining within atherosclerotic brachiocephalic arteries just beyond the junction with the aortic arch from $/ / 1 \mathrm{r} 1^{+/+} \mathrm{Apoe}^{-/-}$and $/ 11 \mathrm{r} 1^{-/-}$ Apoe $^{-/-}$mice. Scale bars: $200 \mu \mathrm{m}$. (B and C) Quantification of the percentage of positive area within the plaque $(\mathbf{B})$ and vessel media (C) for MMP3 from $I / 1 \mathrm{r}^{+/+} \mathrm{Apoe}^{-/-}$and $I / 1 \mathrm{r}^{-l_{-}}$Apoe ${ }^{-/-}$mice. ${ }^{*} P<0.001$ by Mann-Whitney rank sum test. Data in $B$ represent mean \pm SEM $\left(n=14, I / 1 r 1^{+/+} A p o e^{---} ; n=10, \| 11 r^{-l-} A p o e^{-/-}\right)$, and data in $\mathbf{C}$ represent mean $\pm \operatorname{SEM}\left(n=14,\left\|11 r 1^{+/+} A p o e^{-/-} ; n=12,\right\| 11 r 1^{-l-} A_{p o e^{-/-}}\right)$.

vessel wall MMP3 levels in IL-1R1-deficient mice, combined with previous studies demonstrating that MMP3 promotes SMC migration into scratched areas of a SMC monolayer in vitro $(54,55)$, we hypothesized that IL-1 may promote SMC migration through extracellular matrix in an MMP3-dependent manner. Experiments were performed using SMCs from $\mathrm{Mmp}^{3^{+/+}}$and $\mathrm{Mmp}^{3^{-/-}}$mice, which demonstrated that IL-1 significantly increased expression of MMP3 in wild-type SMCs, consistent with previous studies (47); however, in $\mathrm{Mmp3}^{-/-}$SMCs, expression of MMP3 was virtually undetectable with or without IL-1 $\beta$ treatment (Supplemental Figure 7). Using these cells in Transwell invasion assays with a Matrigel barrier of basement membrane matrix, IL-1 $\beta$ promoted migration of $\mathrm{Mmp3}^{+/+} \mathrm{SMCs}$ through Matrigel; however, this effect was abrogated in $\mathrm{Mmp3}^{-/-} \mathrm{SMCs}$ (Figure 5). These results provide evidence that MMP3 plays an important role in promoting IL-1-induced SMC migration through matrix and suggest that the observed reductions in atherosclerotic plaque SMC content and coverage in IL-1R1-deficient mice may be due, at least in part, to decreased MMP3-dependent SMC migration.

\section{Discussion}

The focus of the present studies was to determine the effects of the inflammatory cytokine IL-1 on features of atherosclerotic plaque stability and narrowing of the vessel lumen, both of which are critical in the development of the clinical complications of atherosclerosis (2). Completely contrary to our original hypothesis, results provided surprising evidence that IL-1 promotes multiple features of atherosclerotic plaque stability, including increased plaque SMC content and coverage, reduced intraplaque hemorrhage, and increased plaque collagen content. In addition, the studies provide evidence that IL-1 also promotes outward vessel remodeling to limit reductions in vessel lumen size in advanced atherosclerosis. Finally, these studies demonstrate that IL-1 signaling is a critical regulator of MMP3 expression in advanced atherosclerosis and suggest that MMP3 may be a downstream mediator of IL-1-induced outward vessel remodeling and features of plaque stability, such as increased plaque SMC content and coverage. Taken together, these results suggest that IL-1 plays a dual atheroprotective role in advanced atherosclerosis by promoting outward vessel remodeling and concomitantly enhancing features of plaque stability, perhaps through MMP3-dependent mechanisms.

Results of the present studies demonstrating that inactivation of the lone signaling receptor for the inflammatory cytokine IL-1, IL-1R1, enhanced multiple features of plaque instability are surprising in that multiple proinflammatory cytokines, such as IL-18 (13), IFN- $\gamma$ (15), and monocyte chemoattractant protein 1 (14), have been shown to promote features of atherosclerotic plaque instability in mice, whereas antiinflammatory cytokines IL-10 (56, 


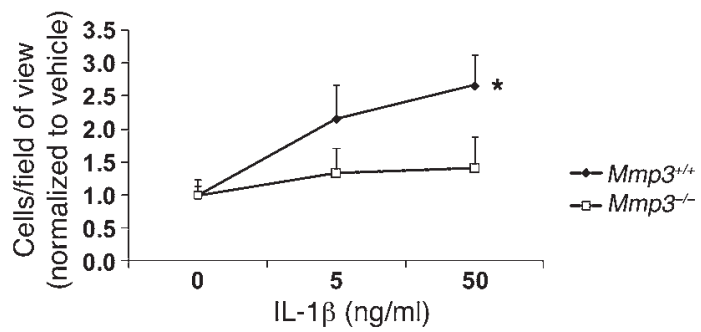

Figure 5

IL-1 $\beta$ promotes MMP3-dependent SMC migration across a Matrigel barrier. SMC number counted on the underside of Matrigel-coated Transwells was increased with IL- $1 \beta$ in the lower chamber in $\mathrm{Mmp3}^{+/+}$ SMCs, but not in $\mathrm{Mmp3}^{-/}$SMCs. ${ }^{*} P<0.05$ for IL-1 $\beta(50 \mathrm{ng} / \mathrm{ml})$ versus vehicle by nested ANOVA. Data represent mean \pm SEM of 3 independent experiments.

$57)$ and TGF- $\beta(58,59)$ reduce features of plaque instability. These results have contributed to the generally accepted dogma that proand antiinflammatory cytokines exert diametrically opposed functions in regulating plaque stability. Our results clearly challenge the notion that proinflammatory cytokines universally promote atherosclerotic plaque destabilization and suggest that IL-1 may play a distinct role relative to other proinflammatory cytokines tested to date, perhaps by promoting profibrotic, reparative aspects of the inflammatory response that may be beneficial in atherosclerosis. Consistent with this, IL-1 has been shown to be particularly profibrotic relative to another classical proinflammatory cytokine, TNF- $\alpha$, in a murine model of pulmonary injury, despite both cytokines inducing similar levels of inflammatory cell infiltration (60). In addition, recent studies have provided evidence that IL-1 can promote macrophage polarization to a profibrotic M2 phenotype (61-63). An important question for future studies is whether the effects of IL-1 that we have observed on features of atherosclerotic plaque stability such as increased collagen content are unique to IL-1 or whether they may extend to other profibrotic inflammatory cytokines that also promote M2 macrophage polarization, such as IL-13 and IL-4 (64-66).

In addition to reducing plaque collagen content, IL-1R1 inactivation reduced features of plaque stability, such as plaque SMC content and coverage. These observations suggest that in the absence of IL-1 signaling, there is a deficit in the ability of SMCs to migrate both into the plaque from the tunica media and to the plaque luminal surface. The marked reductions in MMP3 levels within the plaque and vessel wall of IL-1R1-deficient mice (Figure 4), combined with the observed MMP3 dependence of IL-1 $\beta$-induced SMC migration (Figure 5), suggest that the deficit in SMC content and coverage in IL-1R1-deficient mice may be due to decreased MMP3dependent SMC migration. Although we cannot rule out that other MMPs may also be involved, these findings are consistent with results of previous studies demonstrating decreased SMC content within atherosclerotic plaques of MMP3-deficient Apoe $e^{-/}$mice relative to controls (50). Further studies will be required to determine whether MMP3 expression in SMCs directly mediates the effects of IL-1 to promote increased plaque SMC content and coverage. However, such studies will be extraordinarily difficult and will require conditional, cell-type selective knockout and overexpression of MMP3 in atheroprone mice with and without genetic or pharmacological inactivation of IL-1 signaling.
Previous studies testing the effects of inactivation of IL-1 signaling on atherosclerosis have demonstrated a proatherogenic role for IL-1 in promoting increases in lesion size at the aortic root and increasing the extent of lesion formation in the thoracic aorta $(20$, $22,23,25)$. Interestingly, the only study to date that has tested the effects of inhibition of IL-1 signaling on atherosclerosis at the brachiocephalic artery demonstrated that antibody-mediated inhibition of IL-1 $\beta$ did not significantly alter atherosclerotic plaque size at this site (21). These results are consistent with our observations that IL-1R1 deficiency reduces atherosclerotic plaque size at the aortic root, but not the brachiocephalic artery (Figures 1 and 2). In addition, although we observed consistent decreases in plaque collagen content and plaque SMC coverage as well as consistent increases in intraplaque hemorrhage at the brachiocephalic artery and the aortic root, plaque SMC content was reduced and macrophage content increased at the brachiocephalic artery, whereas plaque SMC content was increased and macrophage content unchanged at the aortic root in IL-1R1-deficient mice (Figure 3 and Supplemental Figure 3). The reasons for these observed differences in the effects of IL-1 on atherosclerosis at the brachiocephalic artery and aortic root are unclear, but may relate to differences arising from the different embryological origins of SMCs at the 2 sites (67) and/or the unique hemodynamics at the aortic root due to the presence of the aortic valves at this site.

Our results demonstrate that inactivation of IL-1 signaling through genetic deficiency of IL-1R1 in advanced murine atherosclerosis results in enhanced features of plaque stability and reduced outward vessel remodeling with increased luminal narrowing. It is possible that at least some of the effects of IL-1R1 deficiency that we have observed, however, may reflect not only loss of IL-1 signaling, but also compensation by other cytokines due to genetic deletion of IL-1R1 over the entire life span of these mice. Indeed, previous studies have observed increased TNF- $\alpha$ production after Klebsiella pneumoniae or influenza virus infection in mice with life-long genetic inactivation of IL-1 signaling $(68,69)$. If such compensatory increases in proinflammatory cytokines have occurred in our IL-1R1-deficient mice, results may not reflect the effects of inhibition of IL-1 in adult mice with a pharmacological agent. Hence, future studies involving administration of pharmacological inhibitors of IL-1 signaling, such as IL-1RN, to mice with advanced atherosclerosis will be important in helping to determine whether acute treatment to reduce but not eliminate IL-1 signaling may decrease features of plaque stability and outward vessel remodeling. If so, additional studies would be important to determine whether acute, localized delivery of IL-1R1 agonists or activation of downstream mediators within atherosclerotic plaques may have protective effects to promote features of plaque stability and outward vessel remodeling.

Our results demonstrating that IL-1R1 deficiency reduces outward remodeling of atherosclerotic brachiocephalic arteries and aortic roots represent, to our knowledge, the first definitive evidence for a causative role for a proinflammatory cytokine in promoting outward vessel remodeling to limit luminal narrowing in spontaneous atherosclerosis. Previous studies have demonstrated increased macrophage content within human atherosclerotic plaques associated with vessels undergoing outward remodeling $(10,70,71)$, suggesting a role for proinflammatory processes in outward vessel remodeling. However, application of the inflammatory cytokines IL-1 $\beta$ and TNF- $\alpha$ to the adventitial surface of normal pig coronary arteries induced neointimal formation 
accompanied by reduced lumen size $(72,73)$, and genetic inactivation of MYD88, the signaling mediator for several inflammatory stimuli, including IL-1, reduced low flow-induced inward vessel remodeling in mice (74). Our results in atherosclerosis, however, suggest that IL-1 actually exerts protective effects to promote outward vessel remodeling and limit reductions in lumen size associated with plaque growth. An important goal for future studies will be to determine whether additional proinflammatory cytokines such as TNF- $\alpha$, IL-17, and IL-18 promote outward remodeling in spontaneous atherosclerosis at the brachiocephalic artery similarly to IL-1 and conversely whether antiinflammatory cytokines may have opposing effects.

Due to the ability of inflammatory cytokines to induce MMP production and release by multiple cell types, it has been thought, although not proven, that inflammatory cytokines promote outward vessel remodeling in atherosclerosis through MMP-mediated matrix degradation and remodeling within the plaque and vessel wall (7). Results of the present studies provide evidence that the matrix metalloproteinase MMP3 promotes outward vessel remodeling in experimental atherosclerosis. These results are consistent with studies of atherosclerosis in humans that showed that MMP3 is selectively increased relative to MMP1, MMP2, and MMP9 in atherosclerotic plaques from outwardly remodeled human coronary arteries (49). Additionally, our results showing that IL-1R1 deficiency was associated with marked and selective reductions in MMP3 levels within atherosclerotic plaques and the associated vessel wall (Figure 4) suggest that MMP3 may mediate the effects of IL-1 to promote outward remodeling of atherosclerotic vessels. Although it is not clear how MMP3 mediates outward vessel remodeling in atherosclerosis, our results demonstrating that IL-1-induced SMC migration is MMP3 dependent suggest that MMP3 may be necessary for SMCs to migrate within the vessel wall to promote matrix remodeling necessary for vessel enlargement (7). As with studies of the role of IL-1 and MMP3 in regulating features of plaque stability, however, determining whether IL-1 promotes outward vessel remodeling in atherosclerosis via MMP3-dependent mechanisms in SMCs will require extensive additional studies, including conditional, SMC-specific MMP3 inactivation in atheroprone mice with excessive and/or deficient IL-1 signaling.

Taken together, results of the present studies provide evidence that IL-1 plays a dual protective role in advanced atherosclerosis through promoting formation of a stable plaque phenotype and concomitantly mediating outward vessel remodeling to limit luminal narrowing. It is, of course, unclear whether results of our studies employing genetic inactivation of the IL-1R1 in atherosclerotic mice will extrapolate to humans; however, our results suggest the potential for unexpected consequences of interrupting atheroprotective effects of IL-1 signaling in rheumatoid arthritis patients receiving IL-1R1 antagonists, such as anakinra (75), or in patients in ongoing clinical trials testing the effects of IL- 1 inhibition on coronary artery disease (28). Additionally, results highlight the general principal that it is overly simplistic to generalize all proinflammatory cytokines as being detrimental for atherosclerotic disease progression and provide evidence of a potential therapeutic benefit to enhancing aspects of the inflammatory response in atherosclerosis. Indeed, our results suggest the need for future studies to explore the potential for diverse roles of different inflammatory cytokines in atherosclerotic plaque stability and outward vessel remodeling in advanced atherosclerosis.

\section{Methods}

Animals. Il1r1 $1^{-/-}$mice (\#003245; Jackson Laboratory), at least 5 generations backcrossed to C57BL/6J (>96\% C57BL/6J), were outcrossed to Apoe $e^{-/-}$mice (\#2052; Jackson Laboratory), which had been backcrossed 11 generations

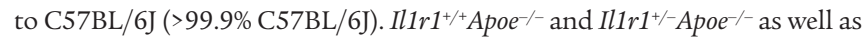

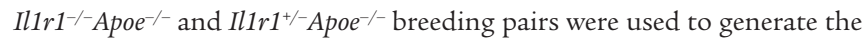
majority of the experimental $I l 1 r 1^{+/} A \mathrm{Poe}^{-/-}$and $\mathrm{Illr1^{+/- }} \mathrm{Apoe}^{-/-}$mice, with 4 experimental mice of each genotype derived from $I l 1 \mathrm{r}^{+/-} \mathrm{Apoe}^{-/-}$intercrosses. Mice were genotyped by PCR according to protocols from the Jackson Laboratory and as described previously (76) using the following primers and conditions: 5'-GAGTTACCCGAGGTCCAGTGG; 5'-CCGAAGAAGCTCACGTTGTCAAG; 5'-GAATGGGCTGACCGCTTCCTCG; $95^{\circ} \mathrm{C}$ for 30 seconds, $65^{\circ} \mathrm{C}$ for 60 seconds, and $72{ }^{\circ} \mathrm{C}$ for 60 seconds.

Animal diet feeding and preparation of tissues. Female $\mathrm{Il1r1^{+/ }} \mathrm{Apoe}^{-/-}$and Illr1 $1^{-/-}$Apoe $e^{-/}$mice were fed a high-fat (Western type) diet containing $21 \%$ milk fat and $0.15 \%$ cholesterol (Harlan Teklad) for 27-30 weeks starting at 8 weeks of age (mean \pm SEM of $28.6 \pm 0.26$ weeks for $I l 1 r 1^{+/+} A p o e^{-/-}$mice and $28.3 \pm 0.27$ weeks for $I l 1 \mathrm{r}^{-/-}$Apoe $e^{-/-}$mice). Mice were sacrificed after a 4-hour fast, and blood was collected after clipping the right atrium. Mice were then perfused via the left ventricle with $5 \mathrm{ml} \mathrm{PBS}$ followed by $10 \mathrm{ml} 4 \%$ paraformaldehyde. Brachiocephalic arteries and hearts containing aortic roots were carefully dissected and fixed overnight in $4 \%$ paraformaldehyde prior to embedding in paraffin. Assays for determining total plasma cholesterol and triglyceride levels (Abbott Laboratories) were performed by the University of Virginia Clinical Pathology Laboratory.

Brachiocephalic artery and aortic root immunohistochemical and morphometric analysis. Paraffin-embedded brachiocephalic arteries were serially sectioned at 5- $\mu \mathrm{m}$ thickness from the aortic arch to the right subclavian artery. For morphometric and immunohistochemical analysis, except where noted below, sections of each brachiocephalic artery were stained at $120-\mu \mathrm{m}$ intervals from 0 to $480 \mu \mathrm{m}$ distal to the aortic arch. Aortic roots were sectioned at $5-\mu \mathrm{m}$ thickness beginning proximally at the first evidence of the aortic valves at their attachment site to the aorta. Sections were stained and analyzed at $150-\mu \mathrm{m}$ intervals from 150 to $600 \mu \mathrm{m}$ distal to this site.

Modified Russell-Movat pentachrome (Movat) staining was performed for morphometric analysis of brachiocephalic arteries and aortic roots (77). For brachiocephalic arteries, areas within the IEL, plaque area, and lumen area were measured directly from the digitized images. At the aortic root, the area within the IEL was calculated using the sum of the lengths of the IEL within each sinus (l) as the circumference of a circle according to the formula $1^{2} / 4 \pi$, and plaque area was subtracted from this value to calculate lumen area, as described previously (32). Picrosirius red staining was performed for analysis of collagen content by measuring birefringence to plane-polarized light $(78,79)$. Antigen Unmasking Solution was used for all immunohistochemistry (Vector Laboratories). Immunohistochemical staining for SM $\alpha$-actin (clone 1A4; Sigma-Aldrich) was performed with detection by Permanent Red (Dako Cytomation). All other immunostaining was detected by DAB (Dako Cytomation). Primary antibodies used with $\mathrm{DAB}$ detection included those raised against the macrophage marker Mac2 (clone M3/38; Accurate Chemical), the rbc marker TER-119 (rat anti-mouse; Santa Cruz Biotechnology Inc.), and the matrix metalloproteinases MMP3 (rabbit anti-human, clone EP1186Y; Epitomics), MMP9 (rabbit anti-rat; Millipore), and MMP13 (goat antimouse; Millipore). Immunostaining for MMP3, MMP9, and MMP13 was performed on tissue sections at the proximal origin of the brachiocephalic artery just beyond the aortic arch. Incubation without primary antibodies and/or with irrelevant species- and isotype-matched immunoglobulins was used as a negative control for all immunostaining.

All image analysis was performed by observers blinded to mouse genotype. Vessel morphometry and areas of positive immunohistochemical 
or picrosirius red staining were quantified using Image-Pro Plus (Media Cybernetics). For detection of positive staining, the portion of the color spectrum representing the positive signal for each stain was defined using the color cube-based method in Image-Pro Plus. This color definition was then applied uniformly to all digitized images for that stain to determine the total positive area within the region of interest such as the plaque or vessel wall, and this positive area measurement was then normalized to the total area of the plaque or vessel wall as described in the text. Plaque SMC coverage was quantified as the percentage of the luminal surface of the plaque covered by SM $\alpha$-actin-positive cells, as described previously (80). Intraplaque hemorrhage was identified as bright red staining within the plaque based on Movat staining with confirmation of the presence of rbc by TER-119 immunohistochemistry. For comparison with the group means, the particular data values for the representative images in Figures 1-4 have been included in Supplemental Table 2.

SMC invasion assays. SMC invasion assays across Matrigel (BD Biosciences) were performed essentially as described (81). Briefly, aortic SMCs isolated from $\mathrm{Mmp}^{\mathrm{3}^{+/+}}$and $\mathrm{Mmp}^{3^{-/-}}$mice (82), a gift from Roger Lijnen (Katholieke Universiteit, Leuven, Belgium), were grown to confluency in $10 \%$ serumcontaining media (DMEM/F12, Gibco, Invitrogen; 10\% FBS, Hyclone; 100 $\mathrm{U} / \mathrm{ml}$ penicillin/streptomycin, Gibco, Invitrogen; $1.6 \mathrm{mmol} / \mathrm{l} \mathrm{L}$-glutamine, Gibco, Invitrogen) and then switched to serum-free medium (DMEM/F12, Gibco, Invitrogen; $1.6 \mathrm{mmol} / \mathrm{l}$ glutamine, Gibco, Invitrogen; $0.2 \mathrm{mmol} / 1$ L-ascorbic acid, Gibco, Invitrogen; $5 \mathrm{mg} / 1$ transferrin, Sigma-Aldrich; 2.8 $\mathrm{mg} / \mathrm{l} \mathrm{recombinant} \mathrm{human} \mathrm{insulin,} \mathrm{Gibco,} \mathrm{Invitrogen;} 100 \mathrm{U} / \mathrm{ml}$ penicillinstreptomycin, Gibco, Invitrogen; $6.25 \mu \mathrm{g} / 1$ selenium, Sigma-Aldrich) for 2 days. Then $20 \mu \mathrm{l}$ of Matrigel (BD Biosciences) at $1 \mathrm{mg} / \mathrm{ml}$ was placed on the upper surface of HTS Transwell Plates containing $8.0-\mu \mathrm{m}$ pores (Corning) for 4 hours at $37^{\circ} \mathrm{C}$. After 4 hours, $2 \times 10^{5}$ cells $/ \mathrm{ml}$ of serum-starved $\mathrm{Mmp}^{+/+}$ or $\mathrm{Mmp3}^{-/-}$SMCs were placed on the upper surface of the Transwells with various doses of recombinant mouse IL- $1 \beta$ (R\&D Systems) or vehicle $(0.1 \%$ BSA) in the lower chamber. After 24 hours, Transwells were washed in PBS, fixed with $10 \%$ formalin, and stained with $0.2 \%$ crystal violet in $7 \%$ ethanol. Upon removing cells from the upper surface of each Transwell, remaining cells were counted in 2 fields of view from the center of each Transwell and the mean of the 2 counts was used as the value for each Transwell.

Statistics. For comparisons of 2 groups of continuous variables with normal distributions (determined by the Kolmogorov-Smirnov test), 2 -tailed Student's $t$ tests were used. For 2-group comparisons from multiple experiments each in triplicate, nested ANOVA was used. Two- group comparisons with nonnormal distributions were analyzed using the MannWhitney rank sum test. Fisher's exact test was used for categorical data. Two-way ANOVA was used for multiple group comparisons, preceded where indicated by $\log _{10}$ or square root transformation of the data to achieve a normal distribution (determined by Kolmogorov-Smirnov test). For data with either nonequal group variances (by the Levene test) or where transformation failed to normalize the data, the Scheirer-Ray-Hare test was performed on ranked data (83). For the Scheirer-Ray-Hare test, randomly generated values from the distribution of the fitted values were added to balance unequal group sample sizes. SigmaStat was used for all statistical tests, with the exception of the Scheirer-Ray-Hare test and nested ANOVA, which were performed using Minitab software (Minitab, Inc.). Only for brachiocephalic artery plaque macrophage content (Figure 3I; $P=0.003$ ), aortic root plaque SMC coverage (Supplemental Figure 3G; $P<0.001$ ), and aortic root plaque SMC content (Supplemental Figure $3 \mathrm{H} ; P<0.001$ ) were significant differences found among locations across the vessels using 2-way ANOVA or Scheirer-Ray-Hare tests; hence, results of location comparisons were otherwise not reported in the manuscript. There were no significant interactions between genotype and location for any of the end points analyzed by the 2 -way ANOVA or Scheirer-Ray-Hare tests. $P<0.05$ was considered significant.

Study approval. Animal protocols used in this study were approved by the Animal Care and Use Committee at the University of Virginia.

\section{Acknowledgments}

This work was supported by NIH grants RO1 HL38854, P01 HL19242, and R01 HL57353 (to G.K. Owens), an American Heart Association predoctoral fellowship (0615326U) and Medical Scientist Training Program training grant (5T32GM007267-26) (to M.R. Alexander), and a Basic Cardiovascular Research training grant (5T32-HL007284) (to C.W. Moehle). The authors would like to thank Dominique Rose, Rupanda Tripathi, Melissa Bevard, and John Sanders for excellent technical assistance; Meera Murgai and Laura Shankman for help with data analysis; Paul Manser and Elizabeth Greene for help with statistical analysis; and Norbert Leitinger, Coleen McNamara, Amy Tucker, and Brian Wamhoff for helpful discussions. We would also like to thank H. Roger Lijnen for the gracious gift of the $\mathrm{Mmp}^{\mathrm{3}^{+/+}}$and $\mathrm{Mmp}^{3^{-/-}}$SMCs.

Received for publication August 23, 2011, and accepted in revised form October 19, 2011.

Address correspondence to: Gary K. Owens, University of Virginia School of Medicine, 415 Lane Road, P.O. Box 801394, Charlottesville, Virginia 22908, USA. Phone: 434.924.2652; Fax: 434.982.0055; E-mail: gko@virginia.edu.
1. Lusis AJ. Atherosclerosis. Nature. 2000 407(6801):233-241.

2. Libby P, Theroux P. Pathophysiology of coronary artery disease. Circulation. 2005;111(25):3481-3488.

3. Galkina E, Ley K. Immune and inflammatory mechanisms of atherosclerosis. Annu Rev Immunol. 2009;27:165-197.

4. Tedgui A, Mallat Z. Cytokines in atherosclerosis: pathogenic and regulatory pathways. Physiol Rev. 2006;86(2):515-581.

5. Libby P, Ridker PM, Hansson GK. Inflammation in atherosclerosis: from pathophysiology to practice. J Am Coll Cardiol. 2009;54(23):2129-2138.

6. Shiran A, et al. Serial volumetric intravascular ultrasound assessment of arterial remodeling in left main coronary artery disease. Am J Cardiol. 1999; 83(10):1427-1432.

7. Pasterkamp G, de Kleijn DPV, Borst C. Arterial remodeling in atherosclerosis, restenosis and after alteration of blood flow: potential mechanisms and clinical implications. Cardiovasc Res. 2000;45(4):843-852.
8. Pasterkamp G, Falk E. Atherosclerotic plaque rupture: an overview. J Clin Basic Cardiol. 2000;3(2):81-86.

9. Ge J, et al. Screening of ruptured plaques in patients with coronary artery disease by intravascular ultrasound. Heart. 1999;81(6):621-627.

10. Burke AP, Kolodgie FD, Farb A, Weber D, Virmani R. Morphological predictors of arterial remodeling in coronary atherosclerosis. Circulation. 2002; 105(3):297-303.

11. Mann JM, Davies MJ. Vulnerable plaque. Relation of characteristics to degree of stenosis in human coronary arteries. Circulation. 1996;94(5):928-931.

12. Kawasaki M, et al. Volumetric quantitative analysis of tissue characteristics of coronary plaques after statin therapy using three-dimensional integrated backscatter intravascular ultrasound. J Am Coll Cardiol. 2005;45(12):1946-1953.

13. Mallat $Z$, et al. Interleukin-18/interleukin-18 binding protein signaling modulates atherosclerotic lesion development and stability. Circ Res. 2001; 89(7):e41-e45.
14. Inoue S, et al. Anti-monocyte chemoattractant protein-1 gene therapy limits progression and destabilization of established atherosclerosis in apolipoprotein E-knockout mice. Circulation. 2002; 106(21):2700-2706

15. Koga M, et al. Inhibition of progression and stabilization of plaques by postnatal interferon-gamma function blocking in ApoE-knockout mice. Circ Res. 2007;101(4):348-356.

16. Dinarello CA. Interleukin-1. Cytokine Growth Factor Rev. 1997;8(4):253-265.

17. Sims JE, Smith DE. The IL-1 family: regulators of immunity. Nat Rev Immunol. 2010;10(2):89-102.

18. Galea J, Armstrong J, Gadsdon P, Holden H, Francis SE, Holt CM. Interleukin-1 beta in coronary arteries of patients with ischemic heart disease. Arterioscler Thromb Vasc Biol. 1996;16(8):1000-1006.

19. Frostegard J, et al. Cytokine expression in advanced human atherosclerotic plaques: dominance of proinflammatory (Th1) and macrophage-stimulating cytokines. Atherosclerosis. 1999;145(1):33-43. 
20. Chamberlain J, et al. Interleukin-1 regulates multiple atherogenic mechanisms in response to fat feeding. PLoS One. 2009;4(4):e5073.

21. Bhaskar $\mathrm{V}$, et al. Monoclonal antibodies targeting IL-1 beta reduce biomarkers of atherosclerosis in vitro and inhibit atherosclerotic plaque formation in Apolipoprotein E-deficient mice. Atherosclerosis. 2011;216(2):313-320

22. Chi H, Messas E, Levine RA, Graves DT, Amar S. Interleukin-1 receptor signaling mediates atherosclerosis associated with bacterial exposure and/or a high-fat diet in a murine apolipoprotein $\mathrm{E}$ heterozygote model: pharmacotherapeutic implications. Circulation. 2004;110(12):1678-1685.

23. Kirii $\mathrm{H}$, et al. Lack of interleukin-1 beta decreases the severity of atherosclerosis in ApoE-deficient mice. Arterioscler Thromb Vasc Biol. 2003;23(4):656-660.

24. Kamari Y, et al. Differential role and tissue specificity of interleukin-1alpha gene expression in atherogenesis and lipid metabolism. Atherosclerosis. 2007; 195(1):31-38.

25. Elhage R, Maret A, Pieraggi MT, Thiers JC, Arnal JF, Bayard F. Differential effects of interleukin-1 receptor antagonist and tumor necrosis factor binding protein on fatty-streak formation in apolipoprotein E-deficient mice. Circulation. 1998;97(3):242-244.

26. Isoda $\mathrm{K}$, et al. Lack of interleukin-1 receptor antagonist modulates plaque composition in apolipoprotein E-deficient mice. Arterioscler Thromb Vasc Biol. 2004;24(6):1068-1073.

27. Banda NK, et al. Intracellular IL-1 receptor antagonist type 1 inhibits IL-1-induced cytokine production in keratinocytes through binding to the third component of the COP9 signalosome. J Immunol. 2005; 174(6):3608-3616.

28. Ridker PM, Thuren T, Zalewski A, Libby P. Interleukin- $1 \beta$ inhibition and the prevention of recurrent cardiovascular events: Rationale and Design of the Canakinumab Anti-inflammatory Thrombosis Outcomes Study (CANTOS). Am Heart J. 2011; 162(4):597-605.

29. Kamari Y, et al. Differential role and tissue specificity of interleukin-1 alpha gene expression in atherogenesis and lipid metabolism. Atherosclerosis. 2007; 195(1):31-38.

30. Rosenfeld ME, Polinsky P, Virmani R, Kauser K, Rubanyi G, Schwartz SM. Advanced atherosclerotic lesions in the innominate artery of the ApoE knockout mouse. Arterioscler Thromb Vasc Biol. 2000;20(12):2587-2592.

31. Williams H, Johnson JL, Carson KGS, Jackson CL. Characteristics of intact and ruptured atherosclerotic plaques in brachiocephalic arteries of apolipoprotein E knockout mice. Arterioscler Thromb Vasc Biol. 2002;22(5):788-792.

32. Bentzon JF, Pasterkamp G, Falk E. Expansive remodeling is a response of the plaque-related vessel wall in aortic roots of apoE-deficient mice: an experiment of nature. Arterioscler Thromb Vasc Biol. 2003; 23(2):257-262

33. Burleigh MC, Briggs AD, Lendon CL, Davies MJ, Born GV, Richardson PD. Collagen types I and III, collagen content, GAGs and mechanical strength of human atherosclerotic plaque caps: span-wise variations. Atherosclerosis. 1992;96(1):71-81.

34. Neumeister V, Scheibe M, Lattke P, Jaross W. Determination of the cholesterol-collagen ratio of arterial atherosclerotic plaques using near infrared spectroscopy as a possible measure of plaque stability. Atherosclerosis. 2002;165(2):251-257.

35. Verhoeven B, et al. Carotid atherosclerotic plaques in patients with transient ischemic attacks and stroke have unstable characteristics compared with plaques in asymptomatic and amaurosis fugax patients. J Vasc Surg. 2005;42(6):1075-1081.

36. Crisby M, Nordin-Fredriksson G, Shah PK, Yano J, Zhu J, Nilsson J. Pravastatin treatment increases collagen content and decreases lipid content, inflammation, metalloproteinases, and cell death in human carotid plaques: implications for plaque stabilization. Circulation. 2001;103(7):926-933.

37. Davies MJ, Richardson PD, Woolf N, Katz DR, Mann J. Risk of thrombosis in human atherosclerotic plaques: role of extracellular lipid, macrophage, and smooth muscle cell content. Br Heart J. 1993;69(5):377-381.

38. van der Wal AC, et al. Clinically stable angina pectoris is not necessarily associated with histologically stable atherosclerotic plaques. Heart. 1996; 76(4):312-316

39. Kolodgie FD, et al. The thin-cap fibroatheroma: a type of vulnerable plaque: the major precursor lesion to acute coronary syndromes. Curr Opin Cardiol. 2001;16(5):285-292.

40. Takaya N, et al. Presence of intraplaque hemorrhage stimulates progression of carotid atherosclerotic plaques: a high-resolution magnetic resonance imaging study. Circulation. 2005;111(21):2768-2775.

41. Kolodgie FD, et al. Intraplaque hemorrhage and progression of coronary atheroma. $N$ Engl J Med. 2003;349(24):2316-2325.

42. Lin HL, et al. Pathological mechanisms and dose dependency of erythrocyte-induced vulnerability of atherosclerotic plaques. J Mol Cell Cardiol. 2007; 43(3):272-280

43. Clarke MC, et al. Chronic apoptosis of vascular smooth muscle cells accelerates atherosclerosis and promotes calcification and medial degeneration. Circ Res. 2008;102(12):1529-1538.

44. Schwartz SM, Virmani R, Rosenfeld ME. The good smooth muscle cells in atherosclerosis. Curr Atheroscler Rep. 2000;2(5):422-429.

45. Clarke MC, et al. Apoptosis of vascular smooth muscle cells induces features of plaque vulnerability in atherosclerosis. Nat Med. 2006;12(9):1075-1080.

46. Newby AC. Do metalloproteinases destabilize vulnerable atherosclerotic plaques? Curr Opin Lipidol. 2006;17(5):556-561.

47. Bond M, Chase AJ, Baker AH, Newby AC. Inhibition of transcription factor NF-kappa B reduces matrix metalloproteinase- $1,-3$, and -9 production by vascular smooth muscle cells. Cardiovasc Res. 2001; 50(3):556-565

48. Sukhova GK, et al. Evidence for increased collagenolysis by interstitial collagenases- 1 and -3 in vulnerable human atheromatous plaques. Circulation. 1999;99(19):2503-2509

49. Schoenhagen P, et al. Relation of matrix-metalloproteinase 3 found in coronary lesion samples retrieved by directional coronary atherectomy to intravascular ultrasound observations on coronary remodeling. Am J Cardiol. 2002;89(12):1354-1359.

50. Johnson JL, George SJ, Newby AC, Jackson CL. Divergent effects of matrix metalloproteinases $3,7,9$, and 12 on atherosclerotic plaque stability in mouse brachiocephalic arteries. Proc Natl Acad Sci U S A. 2005;102(43):15575-15580.

51. Bentzon JF, Weile C, Sondergaard CS, Hindkjaer J, Kassem M, Falk E. Smooth muscle cells in atherosclerosis originate from the local vessel wall and not circulating progenitor cells in ApoE knockout mice. Arterioscler Thromb Vasc Biol. 2006;26(12):2696-2702.

52. Newby AC, Zaltsman AB. Fibrous cap formation or destruction -- the critical importance of vascular smooth muscle cell proliferation, migration and matrix formation. Cardiovasc Res. 1999 41(2):345-360.

53. Maqbool A, et al. MMP-3 (5A/6A) polymorphism does not influence human smooth muscle cell invasion [published online ahead of print April 17 2011]. J Surg Res. doi:10.1016/j.jss.2011.03.043

54. Ugwu F, Lemmens G, Collen D, Lijnen HR. Matrix metalloproteinase deficiencies do not impair cell-associated fibrinolytic activity. Thromb Res. 2001;102(1):61-69.

55. Johnson JL, Dwivedi A, Somerville M, George SJ,
Newby AC. Matrix Metalloproteinase (MMP)-3 activates MMP-9 mediated vascular smooth muscle cell migration and neointima formation in mice. Arterioscler Thromb Vasc Biol. 2011;31(9):e35-e44.

56. Mallat $Z$, et al. Protective role of interleukin-10 in atherosclerosis. Circ Res. 1999;85(8):e17-e24.

57. Potteaux S, et al. Leukocyte-derived interleukin 10 is required for protection against atherosclerosis in low-density lipoprotein receptor knockout mice. Arterioscler Thromb Vasc Biol. 2004;24(8):1474-1478.

58. Lutgens E, et al. Transforming growth factor-beta mediates balance between inflammation and fibrosis during plaque progression. Arterioscler Thromb Vasc Biol. 2002;22(6):975-982.

59. Mallat $Z$, et al. Inhibition of transforming growth factor-beta signaling accelerates atherosclerosis and induces an unstable plaque phenotype in mice. Circ Res. 2001;89(10):930-934.

60. Kolb M, Margetts PJ, Anthony DC, Pitossi F, Gauldie J. Transient expression of IL-1beta induces acute lung injury and chronic repair leading to pulmonary fibrosis. J Clin Invest. 2001;107(12):1529-1536.

61. Triozzi PL, Aldrich W, Singh A. Effects of interleukin-1 receptor antagonist on tumor stroma in experimental uveal melanoma. Invest Ophthalmol Vis Sci. 2011;52(8):5529-5535.

62. Mantovani A, Sica A, Sozzani S, Allavena P, Vecchi A, Locati M. The chemokine system in diverse forms of macrophage activation and polarization. Trends Immunol. 2004;25(12):677-686.

63. Fairweather D, Cihakova D. Alternatively activated macrophages in infection and autoimmunity. J Autoimmun. 2011;33(3-4):222-230.

64. Zhu Z, et al. Pulmonary expression of interleukin-13 causes inflammation, mucus hypersecretion, subepithelial fibrosis, physiologic abnormalities, and eotaxin production. J Clin Invest. 1999;103(6):779-788.

65. Cheever AW, et al. Anti-IL-4 treatment of Schistosoma mansoni-infected mice inhibits development of $\mathrm{T}$ cells and non-B, non-T cells expressing Th2 cytokines while decreasing egg-induced hepatic fibrosis. JImmunol. 1994;153(2):753-759.

66. Wynn TA. Cellular and molecular mechanisms of fibrosis. J Pathol. 2008;214(2):199-210.

67. Majesky MW. Developmental basis of vascular smooth muscle diversity. Arterioscler Thromb Vasc Biol. 2007;27(6):1248-1258.

68. Tanabe M, et al. Compensatory response of IL-1 gene knockout mice after pulmonary infection with Klebsiella pneumoniae. J Med Microbiol. 2005; 54(pt 1):7-13

69. Belisle SE, et al. Genomic profiling of tumor necrosis factor alpha (TNF-alpha) receptor and interleukin-1 receptor knockout mice reveals a link between TNF-alpha signaling and increased severity of 1918 pandemic influenza virus infection. J Virol. 2010;84(24):12576-12588.

70. Pasterkamp G, et al. Relation of arterial geometry to luminal narrowing and histologic markers for plaque vulnerability: the remodeling paradox. $J \mathrm{Am}$ Coll Cardiol. 1998;32(3):655-662.

71. Varnava AM, Mills PG, Davies MJ. Relationship between coronary artery remodeling and plaque vulnerability. Circulation. 2002;105(8):939-943.

72. Shimokawa $\mathrm{H}$, et al. Chronic treatment with interleukin- 1 beta induces coronary intimal lesions and vasospastic responses in pigs in vivo. The role of platelet-derived growth factor. J Clin Invest. 1996; 97(3):769-776

73. Fukumoto $\mathrm{Y}$, et al. Inflammatory cytokines cause coronary arteriosclerosis-like changes and alterations in the smooth-muscle phenotypes in pigs. J Cardiovasc Pharmacol. 1997;29(2):222-231.

74. Tang PC, et al. MyD88-dependent, superoxide-initiated inflammation is necessary for flow-mediated inward remodeling of conduit arteries. J Exp Med. 2008;205(13):3159-3171.

75. Geyer M, Müller-Ladner U. Actual status of anti- 
interleukin-1 therapies in rheumatic diseases. Curr Opin Rheumatol. 2010;22(3):246-251.

76. Palmer G, Talabot-Ayer D, Kaya G, Gabay C. Type I IL-1 receptor mediates IL-1 and intracellular IL-1 receptor antagonist effects in skin inflammation. J Invest Dermatol. 2007;127(8):1938-1946.

77. Prophet EB, Mills SB, Arrington JB, Sobin LH. Afip Laboratory Methods in Histotechnology. Washington, DC, USA: American Registry of Pathology; 1992.

78. Luna LG. Histopathological Methods and Color Atlas of Special Stains and Tissue Artifacts. Gaithersburg, Maryland, USA: American Histolabs; 1992.

79. Junqueira LC, Bignolas G, Brentani RR. Picrosirius staining plus polarization microscopy, a specific method for collagen detection in tissue sections. Histochem J. 1979;11(4):447-455.

80. Lyon CA, Johnson JL, Williams H, Sala-Newby GB, George SJ. Soluble N-cadherin overexpression reduces features of atherosclerotic plaque instability. Arterioscler Thromb Vasc Biol. 2009;29(2):195-201.
81. Kuzuya M, et al. Deficiency of gelatinase a suppresses smooth muscle cell invasion and development of experimental intimal hyperplasia. Circulation. 2003;108(11):1375-1381.

82. Lijnen HR, Silence J, Van Hoef B, Collen D. Stromelysin-1 (MMP-3)-independent gelatinase expression and activation in mice. Blood. 1998;91(6):2045-2053.

83. Sokal R, Rohlf FJ. Biometry: The Principles and Practice of Statistics in Biological Research. 3rd ed. New York, New York, USA: WH Freeman and Company; 1994. 